\title{
Sensory mediation of stimulus-driven attentional capture in multiple-cue displays
}

\author{
RICHARD D. WRIGHT \\ Simon Fraser University, Burnaby, British Columbia, Canada \\ and \\ CHRISTIAN M. RICHARD \\ University of British Columbia, Vancouver, British Columbia, Canada
}

\begin{abstract}
Three location-cuing experiments were conducted in order to examine the stimulus-driven control of attentional capture in multiple-cue displays. These displays consisted of one to four simultaneously presented direct location cues. The results indicated that direct location cuing can produce cue effects that are mediated, in part, by nonattentional processing that occurs simultaneously at multiple locations. When single cues were presented in isolation, however, the resulting cue effect appeared to be due to a combination of sensory processing and attentional capture by the cue. This suggests that the faster responses produced by direct cues may be associated with two different components: an attentionrelated component that can be modulated by goal-driven factors and a nonattentional component that occurs in parallel at multiple direct-cue locations and is minimally affected by goal-driven factors.
\end{abstract}

When viewing a scene, people often perceive a dynamic array of stimulation, with simultaneous changes in scene properties at several different locations. An object at one location, for example, may be moving while an object at another location may be appearing or disappearing. In order to analyze a complex dynamic scene efficiently, one can selectively attend to a subset of its objects or locations. Attentional analysis of visual information is often described in terms of shifts of an attentional focal point to different locations in space in a serial manner. When targets are searched for, these attention shifts can be initiated in response to cues about the probable target locations.

Attention shifts can be initiated by location cues in two ways. Shifts initiated in a goal-driven manner are voluntary and depend on top-down processes. For example, when observers choose to use a cue to direct their attention to an impending target's probable location, this is incorporated into a computational goal for carrying out the task (i.e., the cue information is used to shift attention to the expected target location). Shifts initiated in a stimulusdriven manner, on the other hand, are involuntary under most conditions and appear to depend, in part, on sensory activity generated by the cue's onset. This goal-driven versus stimulus-driven dichotomy has also been referred to as intrinsic versus extrinsic (Milner, 1974), endogenous versus exogenous (Posner, 1978), and voluntary versus

This project was supported by Natural Sciences and Engineering Research Council of Canada Grant 133551, awarded to R.D.W. We are grateful to Lawrence Ward, John McDonald, Marylou Cheal, Jan Theeuwes, Jeremy Wolfe, and an anonymous reviewer for their helpful comments. Address correspondence to R. D. Wright, Department of Psychology, Simon Fraser University, Burnaby, BC, V5A 1S6 Canada (e-mail: rwright@sfu.ca). involuntary (Jonides, 1981; Luria, 1973; Müller \& Rabbitt, 1989).

Symbolic location cues initiate goal-driven attention shifts, and direct location cues initiate stimulus-driven attention shifts. A typical symbolic cue is an arrow or a digit, usually presented in the center of the display, and its meaning can be interpreted by the observer as indicating where the target will probably appear. In contrast, the location information conveyed by a direct cue does not have to be interpreted. Instead, this cue directly indicates a potential target location by virtue of the abrupt luminance change that accompanies its sudden appearance near the location in question. A typical direct cue is an underline, an outline box, or a similar location marker.

The top-down nature of the initiation of attention shifts with symbolic cues is evident if observers discover that the cues are of limited use or if observers are simply told to ignore these cues. In particular, explicit instructions to ignore symbolic cues have been shown to virtually eliminate their effect on responses to targets at cued locations (Jonides, 1981). And when symbolic cues have a low validity (e.g., the probability that the target will appear at the cued location is at chance level or lower), their effect on responses is also attenuated (Jonides, 1981; Kröse \& Julesz, 1989; Müller \& Humphreys, 1991). Presumably, over the course of several trials, observers learn that such cues are not useful for determining the impending target's location, and as a result, the cued location is attended to progressively less often with low-validity symbolic cues. Observers' capacity to voluntarily attend to and process the meaning of symbolic cues indicates that their use as an initiator of attention shifts is top down and goal driven. 
On the other hand, the bottom-up nature of the initiation of attention shifts with direct cues is evident because explicit instructions to ignore these cues does little to attenuate their effect on responses to targets presented at cued locations (Jonides, 1981). And even when direct cues have a low validity, their effect on responses is not attenuated, despite the low probability that a target will appear at these locations (Jonides, 1981; Kröse \& Julesz, 1989; Müller \& Humphreys, 1991). These findings indicate that direct-cue effects on responses to targets at cued locations occur in a bottom-up and stimulus-driven manner that is independent of observers' goals and expectations.

Symbolic location cuing is more closely tied with attentional analysis of the cue than is direct location cuing, because the former is affected to a greater degree by competing demands for attentional resources (see Schneider, Dumais, \& Shiffrin, 1984). In particular, the effectiveness of symbolic and direct cuing was compared when observers were required to identify targets at cued locations, while at the same time, performing a cognitive load task (holding digits in memory) that also required attentional resources (Jonides, 1981). Symbolic-cue effectiveness was progressively diminished by increases in memory load, but direct-cue effectiveness was not. This indicates that in order to use symbolic cues as initiators of attention shifts to expected target locations, attention must be paid to the meaning of these cues. On the other hand, direct cues do not appear to require attentional analysis in order to initiate an attention shift, because their effectiveness is not attenuated by competing demands for attentional resources by the performance of a concurrent task. ${ }^{1}$

Another indication that direct location cue effects depend less on top-down attentional analysis and more on bottom-up sensory analysis is the time course of directcue effectiveness relative to that of symbolic-cue effectiveness. In particular, direct cues usually have their strongest effect on responses to targets at cued locations when the delay between cue and target onsets is $100 \mathrm{msec}$, and when this cue-target onset asynchrony (CTOA) is increased from 100 to $200 \mathrm{msec}$, direct-cue effectiveness is attenuated (Müller \& Findlay, 1988; Müller \& Rabbitt, 1989; Shepherd \& Müller, 1989; Weichselgartner \& Sperling, 1987). In other words, the facilitative effect of direct cues appears to be transient and to last about $200 \mathrm{msec}^{2}$ On the other hand, the effectiveness of symbolic cues appears to increase gradually as the CTOA is increased from 0 to $300 \mathrm{msec}$ and to be sustained at a maximum level with further CTOA increases (e.g., Cheal \& Lyon, 1991; Müller \& Findlay, 1988; Shepherd \& Müller, 1989). Presumably, this is because a period of up to $300 \mathrm{msec}$ is required to selectively attend to and interpret the meaning of symbolic cues. This is markedly different from the transient nature and brevity of directcue effectiveness. The latter suggests that direct cues may facilitate responses to targets at cued locations on the basis of rapid sensory operations triggered by the sudden onsets of these cues.

We have argued elsewhere that when a direct cue appears at a peripheral location $100 \mathrm{msec}$ before the ex- pected target, some visual analysis of the cue must be nonattentional (Wright, Richard, \& McDonald, 1995; Wright \& Ward, 1998). In particular, the location of the cue's onset must be processed before it can provide a signal to the observer about where to shift the attentional focal point. This initial processing is sensory and provides the spatial coordinates required for attentional analysis of the cued location. Note the flaw in the counterargument that attention is required to determine the spatial coordinates of the destination to which attention is to be directed. In order for attention to be directed to a cued location, the spatial coordinates must first be available through nonattentional processing. In other words, the early stages of processing of abrupt-onset visual stimuli such as direct cues are necessarily sensory in nature, and they mediate preattentive localization. Note that we refer to neural activation that is directly triggered by an abrupt change in luminance in the visual scene as sensory processing. ${ }^{3}$ We use this terminology, rather than preattentive processing, because, as is described in the discussion, preattentive operations may also involve a combination of low-level and intermediate-level processes from different stages of visual analysis (e.g., Ullman, 1984).

A number of researchers have argued that the attentional focal point is unitary and not divisible into multiple foci (e.g., Eriksen \& St. James, 1986; Eriksen \& Yeh, 1985; Heinze et al., 1994; Jonides, 1980; Kiefer \& Siple, 1987; McCormick \& Klein, 1990; Posner, Nissen, \& Ogden, 1978; Posner, Snyder, \& Davidson, 1980). This claim is supported by the results of experiments in which symbolic cues indicated two possible target locations and, when asked to attend to both regions, observers were unable to do so effectively (e.g., Kiefer \& Siple, 1987; McCormick \& Klein, 1990). Instead, they sometimes appeared to direct a single attentional focal point to a position midway between the cued locations (Klein $\&$ McCormick, 1989). In other experiments, when distractor stimuli suddenly appeared while attention was focused at another location, the distractors appeared either to "pull" the attentional focal point away from its current position (Müller \& Rabbitt, 1989; Warner, Juola, \& Koshino, 1990) or to "pull" it away only after attention was disengaged (Yantis \& Jonides, 1990). In other words, distractor stimuli in these studies did not appear to cause an attentional focal point to be "split" into multiple foci. Instead, it seemed to remain unitary and indivisible. Note that there is not complete agreement about the unitary attentional focal point assumption, and it continues to be debated in the visual-orienting literature.

In keeping with this assumption, serial attentional analysis has been said to be involved during demanding visual search (e.g., Treisman \& Gormican, 1988) and when inattentional and change blindness occurs (e.g., Mack \& Rock, 1998; Rensink, O’ Regan, \& Clark, 1997, 2000). Others, however, have claimed that demanding visual search could be mediated by parallel processes (e.g., Eckstein, 1998; McElree \& Carrasco, 1999; Mordkoff, Yantis, \& Egeth, 1990), and Palmer has developed a decision-noise parallel model of visual search (Palmer, 
1998; Palmer, Verghese, \& Pavel, 2000). The magnitude of the difficulty involved in determining whether processes mediating visual search operate serially or in parallel is evident when one considers that serial visual search models may actually be a subset of the class of parallel models and that parallel models can be developed to emulate serial search (Bundesen, 1990; Townsend, 1990). Thus, although some experimental results seemed to indicate that serial analysis with a unitary attentional focal point was involved, they may, in fact, have been due to some form of parallel analysis.

One promising way to determine the relative contributions of serial and parallel processes when subjects perform search tasks is the use of functional neuroimaging techniques. Corbetta and colleagues, for example, used PET to show that demanding visual search led to enhanced activity in the posterior parietal cortex (PPC), whereas easy search did not, and that the same area in the PPC is activated when subjects are explicitly instructed to shift their attention from one location to another (Corbetta, Miezin, Shulman, \& Petersen, 1993; Corbetta, Shulman, Miezin, \& Petersen, 1995). Woodman and Luck (2003) used the ERP method to measure N2 (N2-posterior-contralateral) waves during demanding visual search with potential targets in both visual hemifields and found that focused attention appeared to be directed serially to each item, rather than to all the items in parallel. These results indicate that demanding visual search involves at least some degree of serial processing, perhaps with a unitary attentional focal point.

Another paradigm, however, has prompted some researchers to suggest that a unitary attentional focal point can be divided into multiple foci. If two or more direct cues are presented simultaneously, cue effects can occur when targets appear at any of the cued locations (e.g., Richard, Wright, \& Ward, 2003; Wright, 1994; Wright, Richard, \& McDonald, 1996). It could be argued that this occurs because attention has been divided into discrete multiple foci so that it can be directed simultaneously to each cued location (see, e.g., Kramer \& Hahn, 1995). We believe, however, that although some form of parallel processing of cued locations is carried out, it does not involve multiple attentional foci. Instead, as is outlined in this paper, we propose that direct cues trigger a form of sensory processing that can occur in a spatially parallel manner. This processing can influence responses to targets at cued locations, and it can also initiate attentional analysis at single locations in a serial manner.

One proposal that provides a framework for the sensory analysis of simultaneous multiple direct-cue onsets is the activity distribution model (LaBerge, 1998; LaBerge \& Brown, 1989). Sensory activation is said to accumulate in the form of a peaked distribution at the location of each abrupt-onset stimulus. The magnitude of the distribution grows in proportion to the intensity of the stimulus. And because this array of sensory activity is not constrained to a single location at a time, it can change dynamically and in parallel at several locations within a representation of visual space. Attentional analy- sis of information in the representation is said to occur when the magnitude of one peaked activity distribution exceeds a threshold value, thereby causing a channel of focused attention to be opened at its location (see Figure 1). Note that whereas attentional analysis is restricted to only one location at a time, sensory analysis can be carried out at several locations in parallel. And this sensory analysis could mediate preattentive localization of stimuli.

Sensory-based response facilitation is initiated within the framework of this model when the abrupt onset of a direct cue triggers the formation of an activity distribution at its location. Over time, the magnitude of the distribution gradually becomes attenuated until, after $200 \mathrm{msec}$, it disappears. If a second stimulus (the target in question) should appear at roughly the same location before the $200 \mathrm{msec}$ has elapsed, however, another distribution of activity triggered by its abrupt onset may add to any residual activity already at that location as a result of the cue's onset. And their combined activation may cause a channel of attention to be opened up at that location more rapidly than would have been the case if the second stimulus had been presented alone with no prior cuing. Activity distributions can form in parallel at several locations in response to the abrupt onsets of simultaneous multiple direct cues, and so this form of sensorybased response facilitation is not constrained to a single location at any given time.

The present experiments were conducted in order to study the potential contributions of sensory and attentional analyses of targets presented at multiple directcued locations. The goals of this study are not trivial, the implications of the results are not obvious to most researchers, and a theoretical account of multiple-location cuing effects does not already exist in the literature in a well-developed form. To elaborate, it might be suggested that the occurrence of sensory processing of location cues prior to attentional analysis of cues and targets is intuitively apparent. Not all researchers, however, agree about the extent to which the effect of location cues on target-related responses is sensory based. We addressed this question by presenting, in each of the experiments in the study, multiple direct location cues with simultaneous onsets. On the basis of previous findings (e.g., Wright, 1994; Wright et al., 1995), we expected that cue effects would occur at more than one location at a time, thereby indicating that sensory-based processing contributes significantly to target-related responses.

\section{EXPERIMENT 1}

The first experiment was conducted to test directly the idea that sensory processes contribute to the cue effects that occur when target detection tasks are performed. As was mentioned previously, we assume, as do many others, that attention-based cue effects are due to a unitary and indivisible attentional focal point that is directed to only a single continuous region of visual space at any one time. But we suggest that the occurrence of cue ef- 


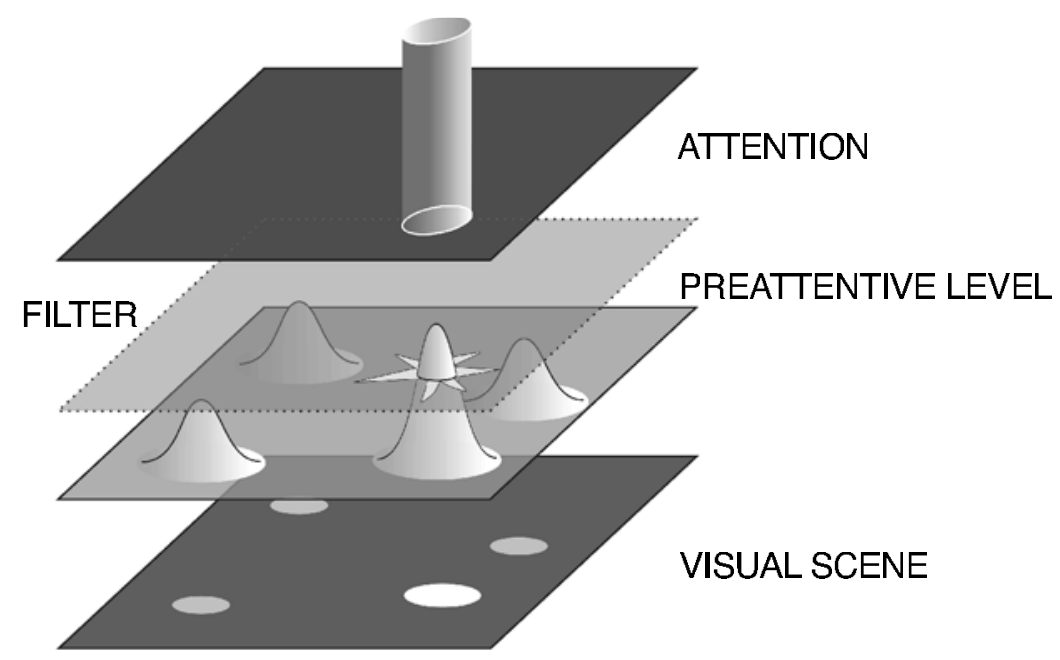

Figure 1. Activity distributions triggered by the onset of simultaneous multiple direct cues. The visual scene corresponds to stimulus displays presented on the computer screen in the present experiments. The preattentive level corresponds to a mental representation of neural activity triggered by changes in sensory inputs. The filter level corresponds to a mechanism proposed by LaBerge (1998) for selecting one of many competing activity distributions for further attentional processing. The attentive level corresponds to a mental representation of the visual scene that the observer is consciously aware of. In this case, one of the distribution magnitudes exceeds the criterion threshold for initiating the opening of a channel of focused attention at that location, and the other three do not. As a result, this location is attended to in a stimulusdriven manner.

fects at more than one location following the simultaneous presentation of multiple direct cues is due to sensory activation triggered by the abrupt onsets of the cues, rather than to a serial analysis of the cued locations with a unitary attentional focal point. In particular, if a target were to appear at any of the cued locations while this sensory activation continued to persist, the time required to detect it might be facilitated. This proposal is markedly different from unitary or multiple attentional focal point accounts of cue effects.

\section{Unitary Attentional Focal Point Account}

The unitary attentional focal point account of simultaneous multiple cuing effects yields a different prediction than does a sensory activation account. By definition, the former is constrained by the assumption that attentional analysis can occur at only one location at a time. Therefore, the unitary focal point account predicts that cue effect magnitudes should change as a function of the number of cues presented. More specifically, if a single cue is presented on a given trial, undivided attentional analysis of it may be possible. But if two cues are presented simultaneously, a unitary and indivisible attentional focal point can be directed to just one of the two cued locations. Averaged over a number of two-cue trials, a cued location would be attended to only $50 \%$ of the time. As a result, the expected cue effect on response times on two-cue trials would be just $50 \%$ of that on single-cue trials, because the mean response time would be based on the $50 \%$ of the trials that yielded an attention-based cue effect plus the $50 \%$ of the trials that yielded no cue effect. Similarly, when averaged over a number of threecue trials, the expected cue effect on response times would be just $33.3 \%$ of that on single-cue trials, because, on average, a cued location would be attended to only $33.3 \%$ of the time. And when averaged over a number of four-cue trials, the expected cue effect on response times would be just $25 \%$ of that on single-cue trials, because, on average, a cued location would be attended to only $25 \%$ of the time. The result is a pattern of cue effect magnitudes that diminishes with increases in cue number.

\section{Multiple Attentional Foci Accounts}

Multiple attentional foci accounts of simultaneous multiple cuing effects also yield different predictions than does a sensory activation account. By definition, the former is not constrained by the assumption that attentional analysis can occur at only one location at a time. Instead, it may be possible to direct an attentional focal point to each of several cued locations. We are unaware of any formal proposal about the maximum number of foci that can utilized at any one time, but presumably any advocates of the multiple attentional foci idea would suggest that there are at least four. This is also the maximum number of cues presented in the present experiments, so the capacity to divide the attentional focal point should, according to proponents of this account, enable one to direct a focus to each of four cued locations. Two variants 
of the multiple foci account of simultaneous multiple cuing effects are that (1) cue effect magnitudes should be equivalent regardless of the number of cues presented, because attentional analysis can occur in parallel at all cued locations with equal effectiveness, or (2) cue effect magnitudes should diminish with increases in cue number (which is similar to the prediction of the unitary attentional focal point account) if subsequent divisions of the attentional focal point lead to progressive decreases in concentration of attentional resources within each focus. In other words, one version of the multiple focal point account holds that there should be no effect of cue number on cue effect magnitudes. The other version holds that increasing the number of foci required to attend to all cued locations at the same time could lead to a decrease in attentional resource concentration within each focus, thereby systematically decreasing cue effect magnitudes.

\section{Sensory Activation Account}

The sensory activation account of simultaneous multiple cuing effects yields yet another prediction. As is the case with the unitary focal point account, it is assumed that attentional analysis can occur at only one location at a time. Therefore, on single-cue trials, attention could be captured by the cue, because there is only one stimulus vying for attention. On multiple-cue trials, however, the sensory activation account holds that initially none of the cued locations would be attended to, because attention cannot be divided among multiple locations. However, sensory activity triggered by the onset of each cue could facilitate the opening of an attention channel when a target subsequently appears at any of the cued locations (if the delay between cue onsets and target onset is brief enough). With one exception, the magnitude of cue effects should be independent of the number of cues presented in the display, because this sensory activation occurs in parallel across the visual scene. The exception is that on single-cue trials, both attentional analysis and sensory activation can contribute to the cue effect, thereby producing a cue effect greater than that on multiple-cue trials. Thus, the sensory activation account predicts the occurrence of cue effects on single-cue and multiple-cue trials, but with the former, the magnitude will be greater.

\section{Method}

Subjects. Twelve Simon Fraser University students were given course credit for taking part in the experiment. All the subjects had normal or corrected-to-normal vision.

Apparatus. A microcomputer (PC) controlled the experiment timing and stimulus presentation. Stimuli were displayed on a 14-in. color monitor, and response times were recorded with a button box interfaced with a dedicated timing board in the computer. The subjects were tested in a dimly lit room in order to minimize reflections, and an adjustable chinrest was used to maintain head position at a distance of $60 \mathrm{~cm}$ from the computer monitor.

Stimuli. All the stimuli were presented on a black (unlit) background. A light gray fixation cross $\left(0.4^{\circ} \times 0.4^{\circ}\right)$ remained visible in the center of the display throughout the experiment. The cues were light gray bars $\left(0.8^{\circ} \times 0.2^{\circ}\right)$, and the target was a bright white line $\left(1.1^{\circ} \times 0.1^{\circ}\right)$ tilted either to the left (on $50 \%$ of the trials) or to the right (on $50 \%$ of the trials) at a $45^{\circ}$ angle. The target was easy to detect under these conditions, thereby reducing the likelihood that the subjects would attempt to execute an eye movement to the target before responding. There were eight possible cue/target positions arranged in a circle around the fixation cross (see Figure 2). The midpoints of all cue locations were $6.2^{\circ}$ from the center of the fixation cross and $5.5^{\circ}$ from the midpoints of adjacent cue locations. The target appeared just above a cue location, so that the cues and the targets did not overlap if both occurred at the same position. This design took into account the results of similar experiments, with the same display and task, that indicated that responses to targets were not biased by (1) greater salience attributable to the "arrow head" configuration seen when the target appeared at a cued location or (2) variation of target location uncertainty as cue number was varied (Richard et al., 2003, Experiments 2C and 3).

Procedure. The subjects were instructed to direct their eyes toward the fixation cross throughout the experiment and to press the response button as quickly as possible when they detected the target's appearance. They were also told that the target was equally likely to appear at a cued or an uncued location on any given trial and, therefore, that cues were uninformative with respect to the potential locations of impending targets.

Each trial began with a 1,000 - to 1,500 -msec intertrial interval. Then one to four cues were presented simultaneously at randomly selected locations and remained visible for the duration of the trial (see Figure 2). Following a second delay of $100 \mathrm{msec}$, the target appeared at one of the eight possible locations and remained visible until the subject responded. Response times were measured as the interval between the target onset and the buttonpress. All the cues and the target were extinguished following the subject's response, which marked the end of the trial. Note that the 100-msec CTOA was short enough to preclude the possibility that the subjects would make eye movements to the cued locations prior to target onset (Fischer \& Weber, 1993) but was long enough to allow direct-cue effects to occur (e.g., Cheal \& Lyon, 1991; Müller \& Findlay, 1988; Shepherd \& Müller, 1989).

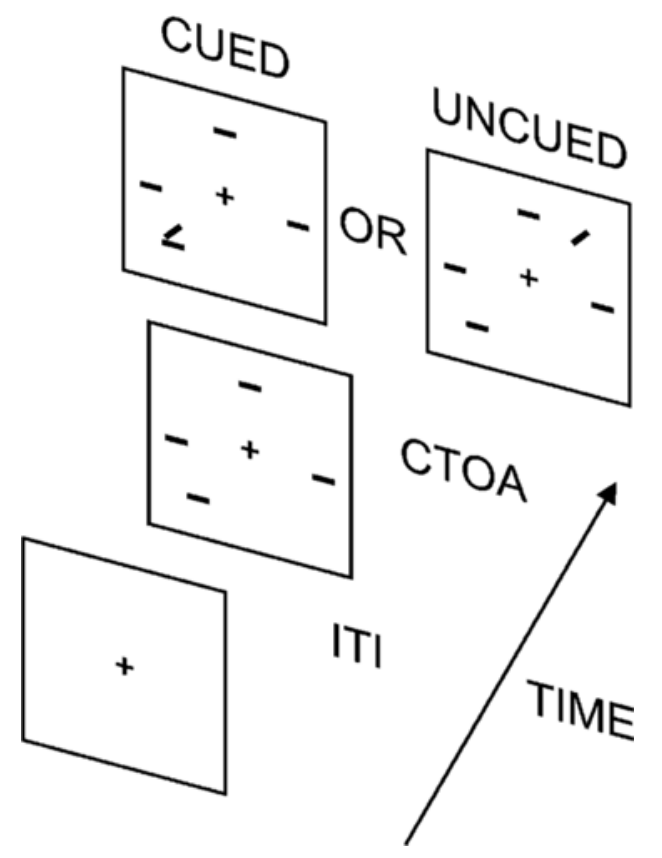

Figure 2. Examples of the type of stimulus displays used in Experiment 1 . Cues were horizontal lines appearing just below potential target locations, and the target was a tilted line. CTOA, cue-target onset asynchrony; ITI, intertrial interval. 
Data were collected in a 1-h testing session, and the subjects were given 30 practice trials before beginning. The session was divided into 20 blocks of 48 data trials. Sixteen catch trials that had 1,500-msec CTOAs before the onset of the target were randomly interspersed in the blocks of trials. Catch-trial responses were collected but not analyzed, because the sole purpose of these trials was to minimize response anticipation errors. A brief rest period followed each block.

Design. The two levels of the target type factor (cued target or uncued target) were completely crossed with the four levels of the cue number factor $(1,2,3$, or 4$)$. Each target type $\times$ cue number combination occurred three times with a left-tilted target and three times with a right-tilted target in each block, and trial presentation order was randomized. The 960 data trials consisted of 120 trials of each combination. The 320 catch trials consisted of 40 trials of each combination.

Data analysis. Before any statistical analyses were carried out, response times less than $100 \mathrm{msec}$ and greater than $1,000 \mathrm{msec}$ were excluded as errors. Following this, response times greater than three standard deviations from the corresponding trial type means were also removed. Analyses of variance (ANOVAs) were conducted with the errors in each experiment to determine whether or not speed-accuracy tradeoffs occurred. These results are not reported in this or other results sections, because no significant tradeoffs occurred in this or the other experiments. The statistical analyses performed in each experiment were repeated measures ANOVAs. Huynh-Feldt corrected degrees of freedom were used to determine probability values for all factors with more than two levels, to compensate for any violation of the assumption of sphericity.

\section{Results and Discussion}

The mean error rate per subject was $4.7 \%$. A $2 \times 4$ repeated measures ANOVA was conducted with the mean response times for each subject in each condition. The main effect of target type was significant $[F(1,11)=61.10$, $\left.M S_{\mathrm{e}}=199.22, p<.001\right]$, indicating that the subjects' response times were shorter when the targets appeared at cued locations than when they appeared at uncued locations. We refer to the difference in mean response times to cued and uncued targets as the cue effect. ${ }^{4}$ The main effect of cue number was also significant $[F(3,33)=$ $\left.5.62, M S_{\mathrm{e}}=77.94, p<.003\right]$. This was due primarily to longer one-cue response times when targets appeared at uncued locations, as opposed to cued locations (see Figure 3 ). In addition, the target type $\times$ cue number interaction was significant $\left[F(3,33)=5.69, M S_{\mathrm{e}}=61.95, p<\right.$ $.003]$. This was also due to longer one-cue response times when the target appeared at uncued locations, as opposed to cued locations.

As can be seen in Figure 3, the cue effect pattern is consistent with the sensory activation proposal. On multiplecue trials, significant cue effects occurred in all the conditions, were smaller than the cue effects on single-cue trials, and did not vary in magnitude as a function of cue number (see Table 1). This pattern is not consistent with either the unitary or the multiple attentional focal point account and suggests instead that cue effects were mediated, in part, by sensory activation. This result also replicates the findings of other simultaneous multiple-location cuing experiments we have conducted (Richard et al., 2003).

A critical assumption of the sensory activation proposal is that cue effects arise from stimulus-driven processing that occurs independently at each cued location. In other words, this processing should be confined to the cued locations and should not spread beyond these regions to encompass nearby uncued locations. In the present experiment, this means that on trials with the target appearing at an uncued location, response times for targets appearing directly between two cued locations should be no different than response times for targets appearing directly between two empty (uncued) locations. If this were not true, response times for uncued targets appearing between two cued locations should more closely match those of cued targets.

In order to confirm that cue effects were restricted to the cued locations, uncued trials were analyzed in terms

\section{EXPERIMENT 1: MEAN RESPONSE TIMES}

\section{EXPERIMENT 1: CUE EFFECTS}
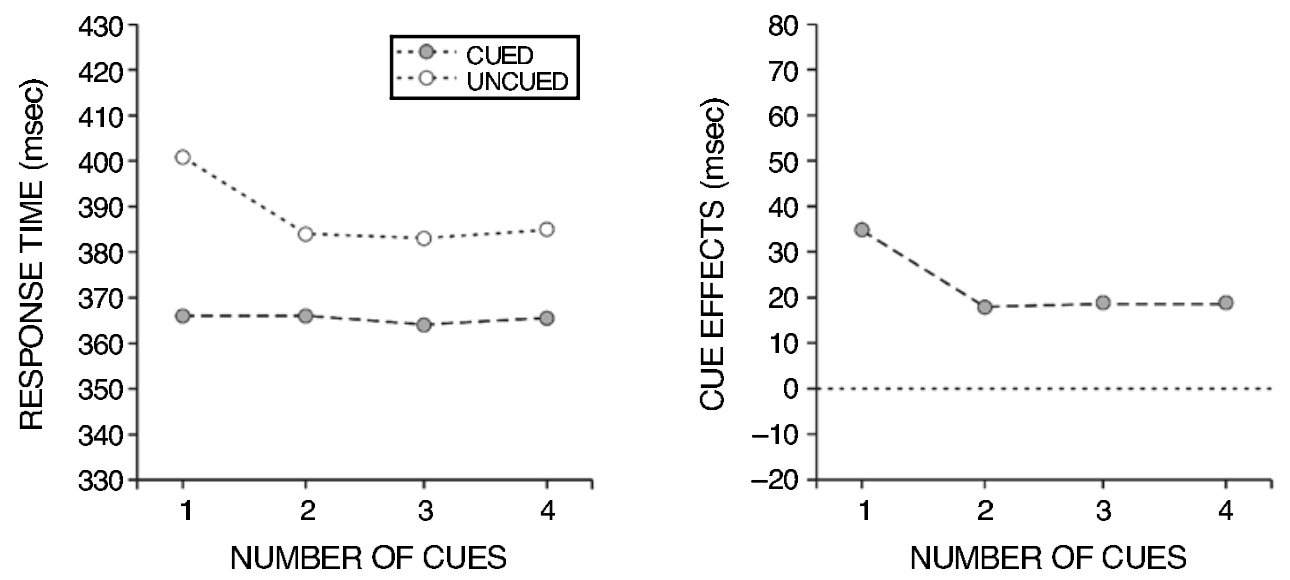

Figure 3. Mean response times and cue effect magnitudes as a function of number of cues presented in Experiment 1. 
Table 1

Mean Cue Effects as a Function of Number of Cues in Experiment 1

\begin{tabular}{ccc}
\hline Number of Cues & Cue Effects & $p$ Value \\
\hline 1 & 35 & $<.001$ \\
2 & 18 & $<.001$ \\
3 & 19 & $<.001$ \\
4 & 19 & .001 \\
\hline
\end{tabular}

of target proximity to cued locations. The proximity category consisted of trials on which the target's location was not immediately adjacent to a cued location. In other words, in the array of eight possible cue/target locations, the positions on either side of the target's location were not occupied by a cue. The proximity ${ }_{1}$ category consisted of trials on which the target was adjacent to a cued location on one side and adjacent to an empty location on

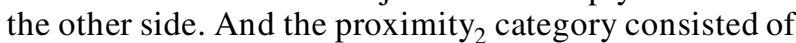
trials on which the target appeared immediately adjacent to two cued locations. The results of this analysis are presented in Table 2..$^{5}$ They indicate that cue-target proximity had no systematic effect on target detection response times in this experiment as a function of cue number and, instead, that cue effects were due to processes that operated independently in a location-specific manner.

Perhaps another unitary attentional focal point account of the equivalence of cue effects on two-cue, threecue, and four-cue trials could be proposed if it is also assumed that the shape of the attentional focus was "warped" to encompass all cued locations, but not the uncued locations between them. This seems unlikely to have occurred because, on many trials in Experiment 1, the attentional focal point would have assumed several irregular "beam" (e.g., banana-like) shapes to wrap around two or more cued locations without encompassing the locations between them. We are not aware of any reported evidence indicating that this is possible, and more important, it is unclear why such a strategy would be adopted to perform the task in Experiment 1, because the impending target's location on a given trial was not related to the cue locations.

A more feasible unitary focal point explanation is that the size of the focused attention was systematically adjusted from one trial to the next to encompass two or more cued locations while leaving some uncued locations outside the attended area. This could produce

Table 2

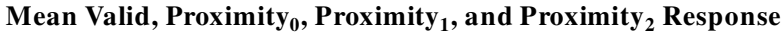
Times (in Milliseconds) and Standard Deviations in

Experiment 1

\begin{tabular}{|c|c|c|c|c|c|c|c|c|}
\hline \multirow{3}{*}{$\begin{array}{l}\text { Number } \\
\text { of Cues }\end{array}$} & \multicolumn{8}{|c|}{ Cue Proximity } \\
\hline & \multicolumn{2}{|c|}{ Valid } & \multicolumn{2}{|c|}{ Proximity $_{0}$} & \multicolumn{2}{|c|}{ Proximity $_{1}$} & \multicolumn{2}{|c|}{ Proximity $_{2}$} \\
\hline & $\mathrm{RT}$ & $S D$ & $\mathrm{RT}$ & $S D$ & $\mathrm{RT}$ & $S D$ & $\mathrm{RT}$ & $S D$ \\
\hline 1 & 366 & 71 & 402 & 81 & 404 & 78 & - & - \\
\hline 2 & 366 & 77 & 392 & 84 & 376 & 87 & - & - \\
\hline 3 & 364 & 75 & 383 & 74 & 383 & 62 & 384 & 81 \\
\hline 4 & 366 & 73 & 390 & 85 & 382 & 79 & 385 & 85 \\
\hline
\end{tabular}

shorter mean cued response times than mean uncued response times. But this proposal is questionable for two reasons. (1) As the cue number increased, the probability that a target would appear outside of the attended area would decrease (because a larger contiguous region of the display area would have to be attended to on trials with more cues). If true, the response time difference between cued and uncued trials should decrease as a function the number of cues presented. As Figure 3 indicates, however, this pattern of diminishing cue effects did not occur. (2) If the attentional focal point encompassed multiple cued locations, uncued locations between cued locations should also have been attended to, and consequently, response times on uncued trials should have been equivalent to response times on cued trials. This prediction was not supported by the proximity analysis. Thus, the pattern of response times observed in Experiment 1 is not consistent with these unitary attentional focal point explanations of the multiple-cue effects.

Note, also, that the pattern of results in Experiment 1 is not consistent with either of the multiple attentional foci predictions. More specifically, response time magnitudes were not equivalent when targets appeared at cued locations, because the cue effects on single-cue trials were greater than those on multiple-cue trials. This suggests that attentional analysis did not occur at all cued locations with equal effectiveness. But if one assumes that the difference in cue effect magnitude on single-cue and multiple-cue trials occurs because dividing the attentional focal point attenuates the concentration of attentional resources within each focus, it follows that subsequent divisions of foci on two-cue, three-cue, and four-cue trials should lead to further attenuations of the concentration of attentional resources within each focus and, therefore, progressively smaller cue effect magnitudes would be predicted as cue number was increased. Therefore, the sensory activation proposal accounts for the data better than does either variant of the multiple attentional foci proposal.

As was mentioned previously, the activity distribution model provides a framework for describing the sensory analysis of simultaneous multiple direct-cue onsets (LaBerge \& Brown, 1989). As can be seen in Figure 1, a peaked activity distribution is said to form in parallel at each cue/target location within a sensory representation. And if the magnitude of a distribution exceeds a relative threshold value, this can cause a channel of focused attention to be opened at the corresponding location within a higher level representation of objects in the visual field. Facilitation of target detection response times can occur in two different ways. (1) If a channel of attention has already been opened at a single cued location, the response time to a target subsequently presented there will be shorter, because the operations involved in opening the attention channel would already be complete. (2) Facilitation can also occur if the delay between cue onset and target onset is brief enough (e.g., $100 \mathrm{msec}$ ) to enable residual activation triggered by the cue to combine with activation triggered by the onset of the target at the 
same location. In this case, the channel of focused attention would be opened more rapidly there than at a target location that was uncued, because the latter does not benefit from the presence of residual activation from a prior cue onset.

According to the sensory activation proposal, attention is captured on single-cue trials when an activity distribution forms in response to the cue's onset and increases in magnitude until it surpasses a threshold value. This, in turn, causes a channel of attention to be opened at that location. On multiple-cue trials, we propose that activity distributions of approximately equal magnitude form at each cued location. None of the distributions would be significantly greater in magnitude than the others, and therefore, none would surpass the relative threshold that causes a channel of attention to be opened at its location. Thus, we propose that the cue effects on multiple-cue trials in Experiment 1 were due to residual sensory activation at all the cued locations that did not capture attention but did enable an attention channel to be opened faster when the target appeared at one of these locations than when the target appeared at an uncued location.

The dynamics of activity distribution formation are also consistent with the occurrence of a larger cue effect in the single-cue condition than in the multiple-cue condition in Experiment 1. As can be seen in Figure 3, this occurred because the mean uncued response time was greater on the single-cue trials than on the multiple-cue trials. According to the sensory activation proposal, the magnitude of an activity distribution at a cued location on a single-cue trial should increase until it surpasses the threshold value for opening a channel of attention there. As a result, the time required to open the attention channel elsewhere if the target appears at an uncued location should increase, because the competing activity distribution triggered by the cue has already begun to initiate the opening of the attention channel at its location. Therefore, the greater mean uncued response time on single-cue trials than on multiple-cue trials reflects an additional cost of attentional disengagement that would not occur on multiple-cue trials.

Note that we chose to display the cues in Experiment 1 until the trial was terminated by the subject's response (i.e., sustained cues). A variant of this procedure would be to present cues for a brief period of time (e.g., $50 \mathrm{msec}$ ) and then remove them prior to the target's onset. It is possible that briefly flashed cues would produce a smaller cue effect than would the sustained cues used in the present experiment. More specifically, in terms of the framework we propose, perhaps activity distributions would dissipate sooner at briefly flashed cue locations than at sustained cue locations. If so, there would be less residual activation present when targets appeared at briefly flashed cue locations than when they appeared at sustained cue locations, and perhaps, a smaller cue effect would be expected with the former. Systematic manipulation of cue duration in a follow-up experiment could, therefore, provide an additional constraint on the sensory activation proposal. ${ }^{6}$

\section{EXPERIMENT 2}

We suggest that the effect of multiple-location cuing on response times in the first experiment was due, in part, to preattentive sensory activation that occurred briefly at each of the cued locations following the cue onsets. The initial, sensory-based neural response to an abrupt luminance change in several brain areas involves a transient increase in activity (e.g., Wurtz \& Albano, 1980). If simultaneous cue effects at multiple locations involve a related type of sensory activity, we would expect cue effects to exhibit a similar transient facilitation pattern in response to an abrupt luminance change, peaking at short CTOAs and declining thereafter (see Müller \& Rabbitt, 1989). On the other hand, if sustained attentional processes are involved, such as the allocating of attentional resources to multiple discrete locations (e.g., Shaw, 1984; Shaw \& Shaw, 1977), we would expect cue effects to remain at elevated levels for several hundred milliseconds (e.g., Hughes, 1984). In this experiment, we manipulated CTOA to determine whether or not the time course of multiple simultaneous direct location cues would produce a transient pattern of cue effects that would be consistent with preattentive sensory activation.

\section{Method}

Subjects. Twelve Simon Fraser University students were given course credit for taking part in the experiment. All the subjects had normal or corrected-to-normal vision.

Stimuli and Procedure. The stimulus displays and procedure were the same as those in Experiment 1, with the following exceptions: Four cues were presented on all the trials, and the CTOA was 100,200 , or $300 \mathrm{msec}$. Note that although $300 \mathrm{msec}$ is sufficient time for executing a saccade (Fischer \& Weber, 1993), using a CTOA of this duration was not a direct concern in the present experiment, because of the impossibility of executing eye movements to four cued locations simultaneously. The testing session was divided into 20 blocks of 36 data trials that were randomly interspersed with 12 catch trials.

Design. The two levels of the target type factor (cued target or uncued target) were completely crossed with the three levels of the CTOA factor $(100,200$, or $300 \mathrm{msec})$. Each target type $\times$ cue number combination occurred three times with a left-tilted target and three times with a right-tilted target in each block, and trial presentation order was randomized. The 720 data trials consisted of 120 trials of each combination. The 240 catch trials consisted of 40 trials of each combination.

\section{Results and Discussion}

The mean error rate per subject was $2.7 \%$. A $2 \times 3$ repeated measures ANOVA was conducted with the mean response times for each subject in each condition. As in the previous experiment, there was a significant main effect of target type $\left[F(1,11)=5.13, M S_{\mathrm{e}}=103.95, p<.05\right]$, because response times were shorter when targets appeared at cued locations than when they appeared at uncued locations. The main effect of CTOA was also significant $\left[F(2,22)=32.96, M S_{\mathrm{e}}=130.96, p<.001\right]$, because response times were shorter when CTOAs were longer. In addition, the target type $\times$ CTOA interaction was significant $\left[F(3,33)=5.69, M S_{\mathrm{e}}=61.95, p<.002\right]$. As can be seen in Figure 4, the difference between responses times 


\section{EXPERIMENT 2: MEAN RESPONSE TIMES}

\section{EXPERIMENT 2: CUE EFFECTS}
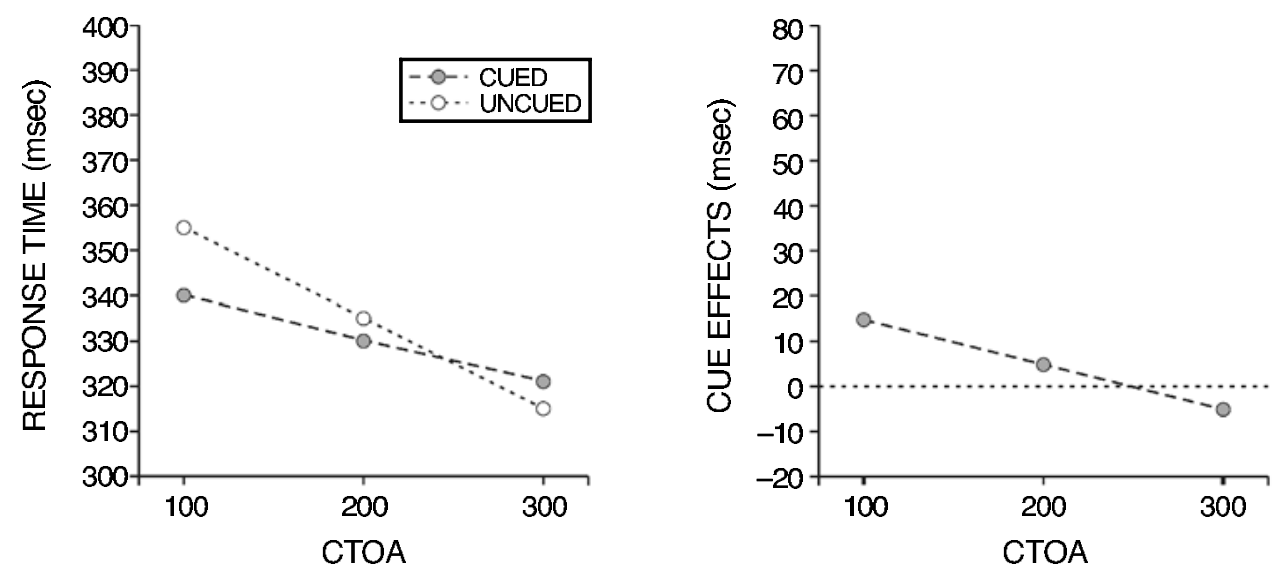

Figure 4. Mean response times and cue effect magnitudes as a function of cue-target onset asynchrony (CTOA) in Experiment 2.

for targets at cued locations and those for targets at uncued locations was significant only when the CTOA was $100 \mathrm{msec}$ (see Table 3 for paired comparisons of means).

These data indicate that multiple-cue effects were maximal when the CTOA was $100 \mathrm{msec}$ and diminished with further increases in CTOA. It could be argued that the cue effect in the 100-msec CTOA condition was due to attentional analysis. Note, however, that the contribution of attentional analysis to cuing effects appears to increase gradually as CTOA is increased from 0 to $300 \mathrm{msec}$ and beyond (e.g., Cheal \& Lyon, 1991; Müller \& Findlay, 1988; Shepherd \& Müller, 1989). Therefore, if attentional analysis led to the cuing effect on $100-\mathrm{msec}$ CTOA trials in Experiment 2, it should have led to cuing effects on 200- and 300-msec CTOA trials as well. The absence of cuing effects in Experiment 2 at the 200-msec CTOA and, particularly, at the 300 -msec CTOA suggests an explanation of the cue effect in the 100-msec CTOA condition that is more consistent with the sensory activation account than with an attentional analysis account.

The sensory activation account of cue effects that we propose is that response times will be facilitated if the CTOA is brief enough to enable any residual cue-triggered activation to combine with target-triggered activation at the same location. This causes the channel of focused attention to be opened more rapidly there than at an uncued location. As is depicted in Figure 5, we suggest that residual cue-triggered activation was maximal at $100 \mathrm{msec}$

Table 3

Mean Cue Effects as a Function of Cue-Target Onset Asynchrony (CTOA) in Experiment 2

\begin{tabular}{ccc}
\hline CTOA $(\mathrm{msec})$ & Cue Effect & $p$ Value \\
\hline 100 & 15 & $<.001$ \\
200 & 5 & .265 \\
300 & -6 & .571 \\
\hline
\end{tabular}

and was sufficient to produce a cue effect at this CTOA in Experiment 2. But on 200- and 300-msec CTOA trials, the residual cue-triggered activation had diminished too much to produce a significant cue effect when the targets were presented at cued locations after this delay.

\section{EXPERIMENT 3}

We suggest that sensory activation mediated the cue effects at up to four locations at the same time in the previous experiments because sensory activation is not constrained to a single location at a time. When CTOA was increased beyond $100 \mathrm{msec}$ in Experiment 2, cue effects were attenuated, as would be expected if they were mediated by sensory activation. In this experiment, we conducted a more direct test of sensory activation's role in mediating cue effects by manipulating cue luminance. The magnitude of sensory activation is directly related to stimulus intensity, and therefore, changes in cue luminance should cause changes in the magnitude of sensory activation triggered by cue onsets. Note that the direct relationship between cue luminance and activation magnitude differs from that between cue luminance and attentionbased cue effects. More specifically, the effect of varying direct-cue luminance was investigated in an experiment that employed highly valid direct cues presented with long CTOAs (Hughes, 1984). The long CTOAs (greater than $100 \mathrm{msec}$ ) provided sufficient time for any potential sensory effects on responses to become attenuated while attentional focus was actively sustained at the cued location. The results indicated that increasing cue luminance enhanced the alerting effect of the cue but did not alter the magnitude of cue effects. We varied cue luminance across trials in the present experiment with noninformative location cues to determine whether or not cue effect magnitudes would change directly as a function of cue luminance. If so, this would not be con- 

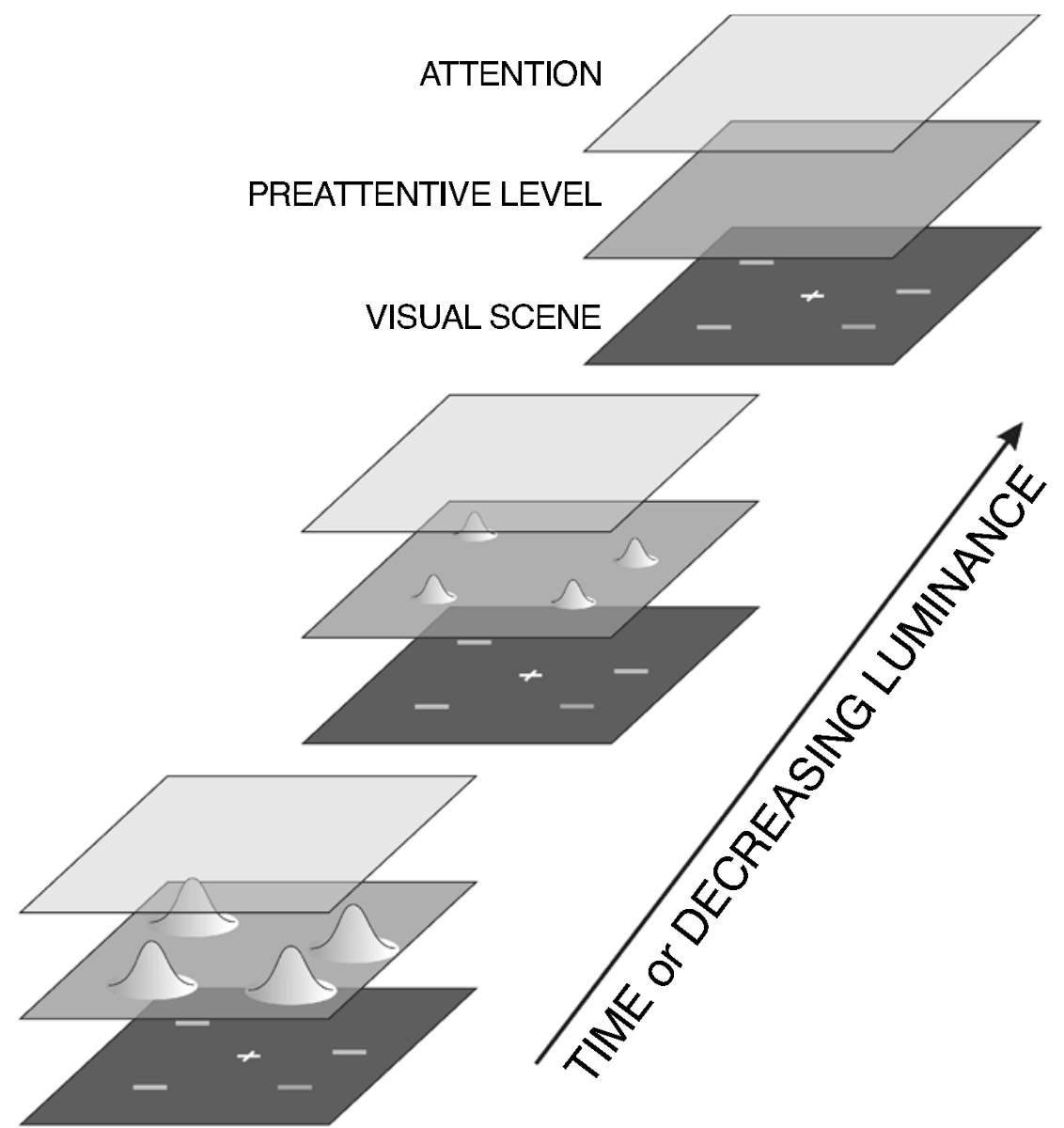

Figure 5. The magnitude of activity distributions diminishes as a function of time since their onset. Thus, in Experiment 2, increases in cue-target onset asynchrony resulted in a decrease in the level of residual activation at each cued location at the time of target onset and, therefore, a decrease in cue effect magnitude. Activity distribution magnitude also corresponds to stimulus intensity. The effect of changes in the luminance of cues on activity distribution magnitudes in Experiment 3 can be represented in this figure by a "decreasing luminance" label.

sistent with previous findings with attention-based tasks. Instead, it would provide further evidence that simultaneous multiple direct-cue effects are mediated, in part, by sensory activation.

\section{Method}

Subjects. Twenty-three Simon Fraser University students were paid $\$ 5$ for participating in this experiment. All the subjects had normal or corrected-to-normal vision.

Stimuli and Procedure. The apparatus and stimuli were the same as those in Experiment 1, except for the following luminance manipulation: Each set of cues presented on a given trial had one of four luminance levels $\left(0.80,1.52,6.29\right.$, or $\left.14.4 \mathrm{~cd} / \mathrm{m}^{2}\right)$. The background luminance of the display was $0.07 \mathrm{~cd} / \mathrm{m}^{2}$, and the luminance of the target was $55.52 \mathrm{~cd} / \mathrm{m}^{2}$. The procedure was also the same as that in Experiment 1, except for the following: Four cues were presented on each trial, and the subjects spent $10 \mathrm{~min}$ in the testing room prior to the experiment in order to adapt their eyes to the dark.

Design. The two levels of the target type factor (cued location or uncued location) were completely crossed with the four levels of the luminance factor. The 720 data trials consisted of 90 cued trials and 90 uncued trials for each of the four luminance levels. The 240 catch trials consisted of 30 trials of each of these combinations.

\section{Results and Discussion}

The data of 2 subjects were excluded from the analysis because error rates exceeded $10 \%$. The mean error rate of the remaining 21 subjects was $2.0 \%$. A $4 \times 2$ repeated measures ANOVA was conducted with the mean response times for all the subjects in each condition. As in the previous experiments, the main effect of target type was significant $\left[F(1,20)=49.95, M S_{\mathrm{e}}=340.83, p<\right.$ $.001]$. And because increases in cue luminance corresponded to decreases in responses times, the main effect of luminance was also significant $[F(3,60)=33.98$, $\left.M S_{\mathrm{e}}=96.78, p<.001\right]$. This is consistent with the notion that increasing cue luminance can enhance the alerting effect of the cues (see Hughes, 1984). In addition, the lu- 
minance $\times$ target type interaction was significant $\left[F(3,60)=18.74, M S_{\mathrm{e}}=72.88, p<.001\right]$, which indicated that the magnitude of the cue effect increased as a function of luminance (see Figure 6). The results of pairwise comparisons indicated that the cue effect was significant at the two higher luminance levels, but not at the two lower luminance levels (see Table 4). The increase in cue effect magnitude with increases in cue luminance is consistent with our claim that sensory activation initiated by cue onsets facilitates target detection response times. Further experiments with more complex designs that include, for example, CTOA $\times$ cue number $\times$ luminance manipulations might also be fruitful.

One property of the activity distribution model is that the magnitudes of distributions are said to grow in proportion to the intensities of the stimuli (LaBerge, 1998). The four different stimulus intensities of the cues presented in this experiment could have produced activity distributions with the range of magnitudes depicted in Figure 6 , but as a function of changes in luminance, rather than in CTOA. And at the two lowest intensities $\left(0.80\right.$ and $\left.1.52 \mathrm{~cd} / \mathrm{m}^{2}\right)$, cue-triggered activation may not have been sufficient to produce a significant cue effect if targets were presented at these locations.

\section{GENERAL DISCUSSION}

The results of these experiments indicate that, following the simultaneous presentation of multiple direct location cues, a cue effect on target detection response times can occur at as many as four locations at the same time. We propose that the results are due to sensory processing being carried out in parallel across the visual scene. Three findings support this. (1) Cue effects in the experiments occurred in parallel but were location specific. The proximity analysis in Experiment 1 indicated that they occurred at cued locations, but not at uncued lo-
Table 4

Mean Cue Effects as a Function of Cue Luminance in Experiment 3

\begin{tabular}{ccc}
\hline Cue Luminance & Cue Effect & $p$ Value \\
\hline 1 & 4 & .129 \\
2 & 6 & .057 \\
3 & 12 & .003 \\
4 & 20 & $<.001$ \\
\hline
\end{tabular}

cations immediately between. (2) Cue effects were transient and attenuated when the delay between cue and target onsets was greater than $100 \mathrm{msec}$, which is consistent with the transient nature of sensory activation by stimuli. (3) Cue effects varied directly as a function of cue luminance. Although it is possible that some other form of parallel analysis model could account for the present data if it satisfied the constraints indicated by these results, we suggest that the sensory activation account is a better fit than either the unitary attentional focal point account or the multiple attentional foci account.

The present results are also similar to those of another study we conducted to differentiate between sensory and attentional contributions to multiple-location cue effects (Richard et al., 2003). In those experiments, we used virtually the same display as that in the four-cue condition of the present experiments, except that one of the cues was unique by virtue of its color. That is, one cue was red, and the other three were gray. Under most conditions, targets were much more likely to occur at the red cue location than at the gray cue locations. This provided the subjects with an incentive to attend to the unique (red) cue location. The results indicated that, although cue effects were associated with all cue locations, they were significantly greater at the unique-cue location. The unique-cue effects were also consistent with what would be expected if observers were making goal-driven
EXPERIMENT 3: MEAN RESPONSE TIMES

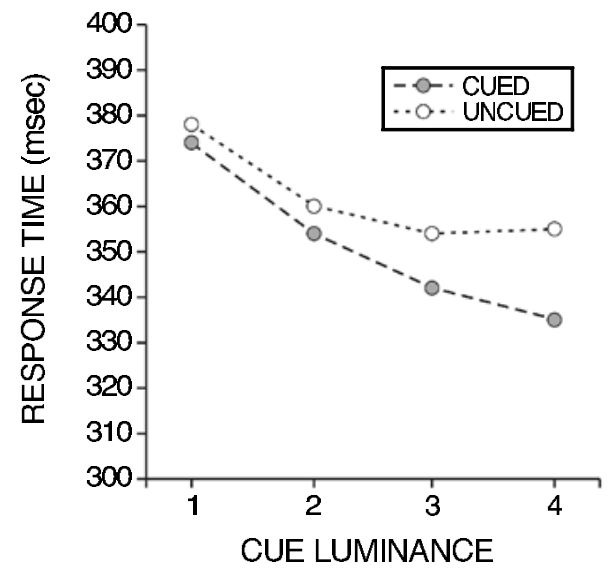

EXPERIMENT 3: CUE EFFECTS

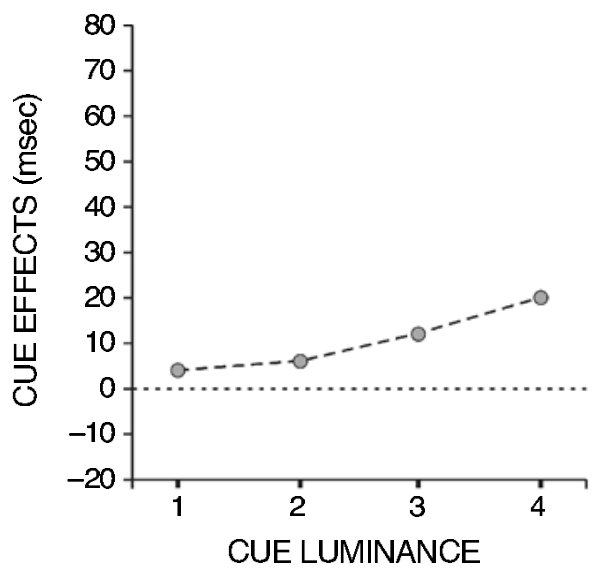

Figure 6. Mean response times and cue effect magnitudes as a function of the luminance of cues presented in Experiment 3. 
attention shifts, because it was sustained over time (as CTOA was increased beyond $100 \mathrm{msec}$ ) but occurred only when cue validity (a priori incentive to attend to the unique-cue location) was high. In contrast, the cue effect at the multiple-cue locations was more transient (diminished at CTOAs greater than $200 \mathrm{msec}$ ) and was not affected by manipulations of cue validity. These findings are consistent with our claim that both sensory and attentional processes underlie single- and multiple-cue effects.

When direct location cue onsets draw attention, the initial stages of their analysis are necessarily sensory and preattentive. Higher level processes that align an attentional focal point with different locations in visual space require information about the spatial coordinates of attention shift destinations before these shifts can be initiated. Logically, this information cannot be supplied by attentional processes themselves, because the purpose of shifting attention to a particular location is to initiate attentional analysis there. To restate and emphasize this point, attentional analysis cannot supply information required for its own initiation. Instead, this information must be supplied by preattentive processes. And it can include information about the locations of direct cues. When described in terms of the sensory activation model, as in Figure 1, preattentive processing involves the formation of an activity distribution that can trigger an attention shift to its corresponding location within a higher level representation of visual space.

One implication of the sensory activation account of the present results is that cued locations are initially encoded preattentively. In particular, when multiple abruptonset stimuli are presented simultaneously, this is said to trigger the formation of activity distributions at each stimulus location within a low-level representation of the visual field. But these locations are not encoded by attentional processes until the magnitude of activation surpasses the relative criterion threshold for opening a channel of focused attention there. That is, the spatial coordinates are available in the lower level sensory representation, but not always in higher level representations that focused attention is aligned within. This is similar to location encoding, said to characterize many other examples of low-level and intermediate-level vision, because much of this analysis involves operations that require information about the relative positions of stimuli in the visual field. Consider, for example, motion correspondence matching (Dawson, 1991; Ullman, 1979). Processing is heavily dependent on location information in order to mediate motion perception in accordance with such rules as the nearest neighbor principle. Stereoscopic fusion of visual images (e.g., Grimson, 1981; Marr \& Poggio, 1977) is another example of processes operating at this level that are heavily dependent on information about the location of elements in the visual field, but this information is often not consciously available to us, because it is determined and utilized by low-level sensory processes. Similarly, the appearance of a location cue object in the visual field will be initially encoded by sensory processes prior to the alignment of focused at- tention at its location. We may not be initially aware of the object's location, and the sensory activation proposal predicts that it will not be attended to in a stimulus-driven manner (i.e., capture attention) until the magnitude of its activity distribution surpasses the criterion threshold.

One ramification of the present results is that simultaneously presented direct location cues should not be used as a neutral cue stimulus for conducting a cost/benefit analysis of valid and invalid cue effects on responses to targets. A neutral cue should provide a warning signal about the impending target's onset that is equal in magnitude to the warning signal provided by the valid and the invalid cues. But neutral cues should not provide any location-specific information about potential target positions. Simultaneous presentation of multiple direct cues has been used as a neutral condition in the past (mistakenly, we argue) because it was assumed at the time that direct cuing effects were entirely the result of attentional analysis. In particular, it was thought that a unitary indivisible focus of attention could not be directed to all cued locations at the same time and, therefore, multiple cuing should be suitable as a neutral cue because perhaps, following their presentation, the observer would not bother to direct attention to any cued location or to just one of several. This assumption does not take into consideration the possibility that (1) direct-cue onsets trigger sensory activation that could also produce a cue effect and (2) the positions of multiple direct cues could be encoded preattentively and provide signals about the impending target's location. We have suggested elsewhere that a more appropriate neutral condition for cost/benefit analysis of direct location cue effects is a uniform flash of the display background (Wright et al., 1995). A flash provides a warning signal about the target's impending onset but does not involve location-specific sensory processing that could produce cue effects, as in the present study.

In summary, the results of the present study indicate that direct location cuing can produce cue effects that are mediated, in part, by sensory processing. And because they are sensory, the effects can occur simultaneously at multiple locations. But when it occurs at a single cued location, the effect appears to be due to a combination of sensory processing and attentional capture by the cue. A model based on LaBerge and Brown's (1989) activity distribution proposal can be used to describe encoding of multiple direct-cue locations and the transient nature of direct-cue effects. The latter may be due to the attenuation of activity distributions prior to target onset. More generally, the results indicate that the effect of direction location cuing on responses to targets may reflect more than simply attentional capture. Preattentive and sensory processing of stimuli also appears to play an important role.

\section{REFERENCES}

Bundesen, C. (1990). A theory of visual attention. Psychological Review, 97, 523-547.

CheAl, M., \& Lyon, D. R. (1991). Central and peripheral precueing of forced-choice discrimination. Quarterly Journal of Experimental Psychology, 43A, 859-880. 
Corbetta, M., Miezin, F. M., Shulman, G. L., \& Petersen, S. E. (1993). A PET study of visuospatial attention. Journal of Neuroscience, 13, 1202-1226.

Corbetta, M., Shulman, G. L., Miezin, F. M., \& Petersen, S. E. (1995). Superior parietal cortex activation during spatial attention shifts and visual feature conjunction. Science, 270, 802-805.

Dawson, M. R. W. (1991). The how and why of what went where in apparent motion: Modeling solutions to the motion correspondence problem. Psychological Review, 98, 569-603.

EcKSTEIN, M. P. (1998). The lower visual search efficiency for conjunctions is due to noise and not serial attentional processing. Psychological Science, 9, 111-118.

ERIKSEN, C. W., \& St. JAmeS, J. D. (1986). Visual attention within and around the field of focal attention: A zoom lens model. Perception \& Psychophysics, 40, 225-240.

ERIKSEN, C. W., \& YEH, Y. (1985). Allocation of attention in the visual field. Journal of Experimental Psychology: Human Perception \& Performance, 11, 583-597.

Fischer, B., \& WEBER, H. (1993). Express saccades and visual attention. Behavioral \& Brain Sciences, 16, 553-610.

Grimson, W. E. L. (1981). From images to surfaces. Cambridge, MA: MIT Press.

Heinze, H.-J., Luck, S. J., Münte, T. F., Gös, A., Mangun, G. R., \& Hillyard, S. A. (1994). Attention to adjacent and separate positions in space: An electrophysiological analysis. Perception \& Psychophysics, 56, 42-52.

Hughes, H. C. (1984). Effects of flash luminance and positional expectancies on visual response latency. Perception \& Psychophysics, 36, 177-184

JonidEs, J. (1980). Towards a model of the mind's eye's movement. Canadian Journal of Psychology, 34, 103-112.

JoNIDES, J. (1981). Voluntary versus automatic control over the mind's eye's movement. In J. B. Long \& A. D. Baddeley (Eds.), Attention and performance IX (pp. 187-203). Hillsdale, NJ: Erlbaum.

Kiefer, R. J., \& SiPLe, P. (1987). Spatial constraints on the voluntary control of attention across visual space. Canadian Journal of Psychology, 41, 474-489.

KLEIN, R, \& McCormick, P. A. (1989). Covert visual orienting: Hemifield activation can be mimicked by zoom lens and mid-location placement strategies. Acta Psychologica, 70, 235-250.

Kramer, A. F., \& HAhN, S. (1995). Splitting the beam: Distributions of attention over noncontiguous regions of the visual field. Psychological Science, 6, 381-385.

KRöSE, B. J. A., \& Julesz, B. (1989). The control and speed of shifts of attention. Vision Research, 29, 1607-1619.

LaBerge, D. (1998). Attentional emphasis in visual orienting and resolving. In R. D. Wright (Ed.), Visual attention (pp. 417-454). New York: Oxford University Press.

LaBerge, D., \& Brown, V. (1989). Theory of attentional operations in shape identification. Psychological Review, 96, 101-124.

LuRIA, A. R. (1973). The working brain. New York: Penguin.

MAck, A., \& Rock, I. (1998). Inattentionalblindness. Cambridge, MA: MIT Press.

Marr, D., \& Poggio, T. (1977). Cooperative computation of stereo disparity. Science, 195, 283-287.

McCormick, P. A., \& KLEIN, R. (1990). The spatial distribution of attention during covert visual orienting. Acta Psychologica, 75, 225-242.

McElree, B., \& Carrasco, M. (1999). The temporal dynamics of visual search: Evidence for parallel processing in feature and conjunction searches. Journal of Experimental Psychology: Human Perception \& Performance, 25, 1517-1539.

Milner, P. (1974). A model for visual shape recognition. Psychological Review, 81, 521-535.

Mordkoff, J. T., Yantis, S., \& Egeth, H. E. (1990). Detecting conjunctions of color and form in parallel. Perception \& Psychophysics, 48, 157-168.

MÜller, H. J., \& Findlay, J. M. (1988). The effect of visual attention on peripheral discrimination thresholds in single and multiple element displays. Acta Psychologica, 69, 129-155.

Müller, H. J., \& Humphreys, G. W. (1991). Luminance-increment de- tection: Capacity-limited or not? Journal of Experimental Psychology: Human Perception \& Performance, 17, 107-124.

Müller, H. J., \& RAbBitt, P. M. A. (1989). Reflexive and voluntary orienting of visual attention: Time course activation and resistance to interruption. Journal of Experimental Psychology: Human Perception \& Performance, 15, 315-330.

Palmer, J. (1998). Attentional effects in visual search: Relating search accuracy and search time. In R. D. Wright (Ed.), Visual attention (pp. 348-388). New York: Oxford University Press.

Palmer, J., Verghese, P., \& Pavel, M. (2000). The psychophysics of visual search. Vision Research, 40, 1227-1268.

Posner, M. I. (1978). Chronometric explorations of mind. Hillsdale, NJ: Erlbaum.

Posner, M. I., Nissen, M. J., \& Ogden, W. C. (1978). Attended and unattended processing modes: The role of set for spatial location. In H. L. Pick \& I. J. Saltzman (Eds.), Modes of perceiving and processing information (pp. 137-157). Hillsdale, NJ: Erlbaum.

Posner, M. I., Snyder, C. R. R., \& Davidson, B. J. (1980). Attention and the detection of signals. Journal of Experimental Psychology: General, 109, 160-174.

Rensink, R. A., O'Regan, J. K., \& Clark, J. J. (1997). To see or not to see: The need for attention to perceive changes in scenes. Psychological Science, 8, 368-373.

Rensink, R. A., O'Regan, J. K., \& Clark, J. J. (2000). On the failure to detect changes in scenes across brief interruptions. Visual Cognition, 7, 127-145.

Richard, C. M., Wright, R. D., \& WARD, L. M. (2003). Goal-driven modulation of stimulus-driven attentional capture in multiple-cue displays. Perception \& Psychophysics, 65, 939-955.

SchNeider, W., Dumais, S. T., \& Shiffrin, R. M. (1984). Automatic and controlled processing and attention. In R. Parasuraman \& D. R. Davies (Eds.), Varieties of attention (pp. 1-27). Orlando, FL: Academic Press.

SHAw, M. L. (1984). Division of attention among spatial locations: A fundamental difference between detection of letters and detection of luminance increments. In H. Bouma \& D. G. Bouwhuis (Eds.), Attention and performance $X$ (pp. 109-121). Hillsdale, NJ: Erlbaum.

Shaw, M. L., \& SHaw, P. (1977). Optimal allocation of cognitive resources to spatial locations. Journal of Experimental Psychology: Human Perception \& Performance, 3, 201-211.

Shepherd, M., \& Müller, H. J. (1989). Movement versus focusing of visual attention. Perception \& Psychophysics, 46, 146-154.

TownsEnd, J. T. (1990). Serial vs. parallel processing: Sometimes they look like Tweedledum and Tweedledee but they can (and should) be distinguished. Psychological Science, 1, 46-54.

Treisman, A., \& Gormican, S. (1988). Feature analysis in early vision: Evidence from search asymmetries. Psychological Review, 95, 15-48. Ullman, S. (1979). The interpretation of visual motion. Cambridge, MA: MIT Press.

Ullman, S. (1984). Visual routines. Cognition, 18, 97-159.

Warner, C. B., Juola, J. F., \& Koshino, H. (1990). Voluntary allocation versus automatic capture of visual attention. Perception \& Psychophysics, 48, 243-251.

Weichselgartner,E, \& Sperling, G. (1987). Dynamics of automatic and controlled visual attention. Science, 238, 778-780.

Woodman, G. F., \& Luck, S. J. (2003). Serial deployment of attention during visual search. Journal of Experimental Psychology: Human Perception \& Performance, 29, 121-138.

WRIGHT, R. D. (1994). Shifts of visual attention to multiple simultaneous location cues. Canadian Journal of Experimental Psychology, 48, 205-217.

Wright, R. D., Richard, C. M., \& McDonald, J. J. (1995). Neutral location cues and cost/benefit analysis of visual attention shifts. Canadian Journal of Experimental Psychology, 49, 540-548.

Wright, R. D., Richard, C. M., \& McDonald, J. J. (1996, June). Simultaneous sensory-driven effects of cue onset at multiple locations in visual space. Paper presented at the annual meeting of the Canadian Society for Brain, Behaviour, and Cognitive Science, Montreal. Wright, R. D., \& WARD, L. M. (1998). The control of visual attention. In R. D. Wright (Ed.), Visual attention (pp. 132-186). New York: Oxford University Press. 
Wurtz, R. H., \& Albano, J. E. (1980). Visual-motor function of the primate superior colliculus. Annual Review of Neuroscience, 3, 189-226.

YANTIS, S., \& JonidES, J. (1990). Abrupt visual onsets and selective attention: Voluntary versus automatic allocation. Journal of Experimental Psychology: Human Perception \& Performance, 16, 121134

\section{NOTES}

1. It is tempting to suggest that direct cuing triggers involuntary shifts of attention to cued locations because these shifts are stimulus driven and their initiation occurs independently of attentional analysis. But this apparent involuntary nature should be qualified. In particular, it appears that although direct cues can trigger shifts of attention to the locations of their onsets, this can be overridden if observers actively focus attention elsewhere at the time of the cues' appearance (e.g., Yantis \& Jonides, 1990). In other words, an attention shift is not a mandatory consequence of a direct-cue onset. Without this capacity to suppress attention shifts to stimuli that suddenly appear in our visual field, we would often be distracted by irrelevant visual events, and it would be difficult to perform tasks requiring sustained attention and vigilance.

2 . In some cases, the effectiveness of direct cues can be sustained at a level slightly lower than the maximum level with CTOAs longer than
$100 \mathrm{msec}$ (Cheal \& Lyon, 1991). But this sustained direct-cue effect at these longer CTOAs appears to occur only if the cue also continues to function as a symbolic cue after the initial reflexive effects of its abrupt onset subside. Also, as was pointed out by Marylou Cheal, when target identification tasks (e.g., color or orientation identification) are performed, direct-cue effectiveness appears to be maximal at CTOAs even shorter than $100 \mathrm{msec}$.

3. It is also possible that other visual events, such as stimulus offsets and transient intensity changes, could trigger sensory processing that mediates preattentive localization.

4. The term cue effect refers to the difference between mean invalid and mean valid response times. It is a general measure of the effect of location cuing on target detection and identification response times, and because it is calculated without a neutral baseline, it is equivalent to the sum of the cost and the benefit of cuing.

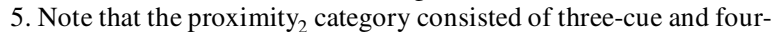
cue trials. The small number of one-cue (not possible) and two-cue trials in this category did not provide enough data points for these trials to be analyzed.

6. We thank Jan Theeuwes for this insight.

(Manuscript received May 18, 2000; revision accepted for publication March 18, 2003.) 